\title{
Synthesis of Porous Confined lonic Liquid Phosphotungstate Based on MIL-101 and Its Solvent-Free Catalytic Oxidation of Cyclohexene to Adipic Acid
}

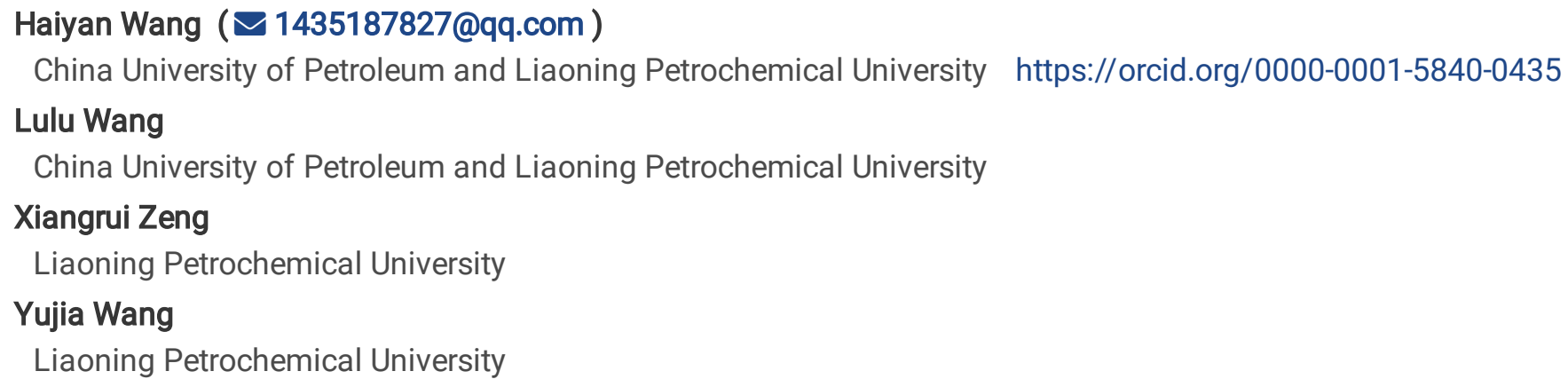

\section{Research Article}

Keywords: Metal-organic frameworks, Cyclohexene, Adipic acid, Catalyst, Heteropolyanion-based lonic Liquids

Posted Date: April 1st, 2021

DOI: https://doi.org/10.21203/rs.3.rs-366501/v1

License: (c) (i) This work is licensed under a Creative Commons Attribution 4.0 International License. Read Full License 


\section{Abstract}

A series of porous confined ionic liquid phosphotungstate were synthesized by encapsulated of 1-(3sulfopropyl)morpholine phosphotungstate $\left(\left[\mathrm{C}_{3} \mathrm{SO}_{3} \mathrm{Hnhm}\right]_{3}-\mathrm{PW}_{12} \mathrm{O}_{40}\right)$ into MIL-101. Then the aforementioned porous confined ionic liquid phosphotungstate as heterogeneous catalyst will be used together with $\mathrm{H}_{2} \mathrm{O}_{2}$ for the catalytic synthesis of adipic acid from cyclohexene. During the experiment, the effect of weight content of $\left[\mathrm{C}_{3} \mathrm{SO}_{3} \mathrm{Hnhm}_{3} \mathrm{PW}_{12} \mathrm{O}_{40}\right.$ on the catalytic performance was investigated in detail. And the chemical structure of the catalyst was also characterized by XRD, FT-IR, SEM-EDX and $\mathrm{N}_{2}$ adsorption and desorption isotherms. Experimental results revealed that the desired products has been successfully prepared, and the best catalytic performance (81.2\% yield of the adipic acid) was obtained under the dose of $\left[\mathrm{C}_{3} \mathrm{SO}_{3} \mathrm{Hnhm}\right]_{3} \mathrm{PW}_{12} \mathrm{O}_{40}$ was $31 \mathrm{wt} \%$, which was almost close to the catalytic activity of original $\left[\mathrm{C}_{3} \mathrm{SO}_{3} \mathrm{Hnhm}\right]_{3} \mathrm{PW}_{12} \mathrm{O}_{40}$ (85.3\% yield of the adipic acid), which was also proved the strategy of packaging ionic liquid phosphotungstate with MIL-101 in this research is feasible. Furthermore, recycled catalytic performance of the catalyst under repeat 6 times was evaluated and the result revealed that the yield in adipic acid was still higher than $80.0 \%$, which also proved that the catalytically active substance was stable in the catalyst.

\section{Introduction}

Adipic acid (AA), as one of the most important dibasic acids in aliphatic acid, is a high value-added fine chemical product, which is widely used in the fields of nylon 66 synthetic, environmentally friendly plasticizers, food additives, pharmaceutical intermediates, etc ${ }^{1]}$. In modern industrial production of AA, a nitric acid oxidation method by using a mixture of cyclohexanol and cyclohexanone as raw materials or a cyclohexane two-step oxidation method is usually adopted [2, $\left.{ }^{3}\right]$. Although the yield of AA and selectivity of these two aforementioned processes are relatively considerable. However, no matter which one of the above two methods, which the equipment will be severely corroded. Moreover, the nitric acid oxidation emits greenhouse gas $\mathrm{N}_{2} \mathrm{O}$, which leads to the greenhouse effect and global warming, which seriously damages the ecological environment $[4,5]$. Therefore, it is imperative to develop an environmentally friendly AA synthesis route.

In recent years, a great deal of research on the novel catalyst of catalytic reaction of cyclohexene to product AA has been reported, which was due to the short and clean process route and low investment cost. Sato et al. ${ }^{6]}$ first developed a clean production process of AA with direct cyclohexene oxidation by $\mathrm{H}_{2} \mathrm{O}_{2}$ in the existence of $\mathrm{Na}_{2} \mathrm{WO}_{4}$ as the catalyst in 1998 . Subsequently, various tungstate-based homogeneous catalysts also have been reported and applied to catalytic the production of cyclohexene to $A A$, which these aforementioned catalysts included that $\left(\mathrm{H}_{2} \mathrm{en}\right)_{3}\left[\mathrm{P}_{2} \mathrm{~W}_{18} \mathrm{O}_{62}\right]_{6} \cdot{ }_{6} \cdot 48 \mathrm{H}_{2} \mathrm{O}\left[{ }^{7]}\right.$, $\mathrm{WO}\left(\mathrm{O}_{2}\right)_{2} \cdot 2 \mathrm{QOH}\left[{ }^{8}\right], \mathrm{H}_{3} \mathrm{PW}_{12} \mathrm{O}_{40}\left[{ }^{9]}\right.$, and these catalytic performance results showed the remarkable catalytic activity of tungstate for oxidation of cyclohexene. However, owing to the good solubility of these homogeneous catalysts in water result in the catalyst was difficult to separate and poor reusability. Eventhough, a lot of heteropolysalts such as $\left.\mathrm{N}\left(\mathrm{C}_{3} \mathrm{H}_{7}\right)_{4}\right)_{3} \mathrm{PMo}_{12} \mathrm{O}_{40},\left(\mathrm{NH}_{4}\right)_{3} \mathrm{PMo}_{12} \mathrm{O}_{40}, \mathrm{Cs}_{3} \mathrm{PMo}_{12} \mathrm{O}_{40}, \mathrm{Cs}_{3} \mathrm{PW}_{12} \mathrm{O}_{40}\left[{ }^{10]}, \mathrm{K}_{3} \mathrm{PMo}_{12} \mathrm{O}_{40}, \mathrm{Cs}_{3} \mathrm{PMo}_{12} \mathrm{O}_{40}, \mathrm{~K}_{3} \mathrm{PW}_{12} \mathrm{O}_{40}\left[{ }^{[1]}\right.\right.$, which can alleviate the aforementioned problems of difficult separation and poor reusability. However, the heterogeneous catalytic process needs large number of acidic or polar solvent to achieve the high yield of AA, and the solvent is always costly and need to be separated from the product. In addition, it has been found that the addition of the acid such as acetic acid[11], hydrochloric acid[ ${ }^{12]}$, acidic ionic liquids[ ${ }^{13]}$ into the polyoxometalates (POMs) can helps to increase the yield of $A A$, because the acid additive creates the acidic media, which the POMs based tungstate was oxidized under the acidic media by $\mathrm{H}_{2} \mathrm{O}_{2}$ and then formed the peroxotungstate species $\left[{ }^{14]}\right.$, which was avail to increase the catalytic activity for oxidation of cyclohexene. The dispersion of POMs on solid supports with a high surface area, is useful for increasing the catalytic activity. Several supports $\left(\mathrm{SiO}_{2}\left[{ }^{15]}, \mathrm{TiO}_{2}\left[{ }^{[6]}, \mathrm{ZrO}_{2}\left[{ }^{[7]}, \mathrm{CeO}_{2}\left[{ }^{18]}, \mathrm{SPC}\left[{ }^{19]}\right)\right.\right.\right.\right.\right.$ have been widely used to disperse POMs. However, heteropolyacid or heteropolysalts dispersed on the support is easily leached into solution, especially it will be more seriously in polar solvent. Therefore, further large-scale promotion and use of the aforementioned catalyst are restricted by the leaching of ionic liquids or acid, which was loaded in the catalyst and high cost of the catalyst.

Page 2/19 
Metal organic framework (MOF) material is a porous crystalline material formed by self-assembly of organic ligands and metal ions $\left[{ }^{20}\right]$, which its regular pore structure, large specific surface area and high porosity are more suitable as the carrier material than other existing porous materials $\left[{ }^{21}\right]$. As one of the typical MOFs, MIL-101 can stably exist in various solvents and its structure can be maintained intact even at a high temperature of $300^{\circ} \mathrm{C}$.

Considering that MIL-101 has the aforementioned advantages and the adjustable pore structure[22], thus in this research, we designed a novel catalyst based on the MIL-101, which was carried on the synthesis of MIL-101 by in-situ hydrothermal method, and during the synchronize synthesis process the sulfonic acid functionalized ionic liquid tungsten phosphotungsten was also encapsulated into the structure of MIL-101. The size effect will ensure that the bigger active component cannot go through the window of MIL-101, thereby it can avoid the problem of easy loss of conventional loaded active components, that is, molecules smaller than the window of the reaction substrate (cyclohexene, $0.42 \mathrm{~nm}$ ) can enter the channel to reach the active center, but molecules larger than the window cannot enter. In other words, the regular and uniform pores of MIL-101 will become a nanocage reactor, and the reaction substrate can fully contact with the active components. On the other hand, the hollow octahedron structure of MIL-101 in micron level can be easily recovered by centrifugation from the reactant. Then, the aforementioned designed hollow octahedron of MIL-101 loaded with ionic liquid phosphotungstate will be used in the cyclohexene catalytic oxidation, which the aforementioned catalyst anticipated showing excellent catalytic activity, substrate molecular size selectivity and better reusability.

\section{Experimental}

\subsection{Materials and reagents}

Morpholine, phosphotungstic acid, 1, 3-propane sultone, acetone and cyclohexene procured from Sinopharm Chemical Reagent Co., Ltd. Chromium(III) nitrate nonahydrate (99.0\%), terephthalic acid (99.0\%), hydrofluoric acid, N, N-dimethyl formamide (DMF), ethanol and hydrogen peroxide (30\%) were obtained from Aladdin Industrial Co., Ltd. (Shanghai, China). The above chemical reagents are all of analytical grade. The deionized water was used throughout in this research.

\subsection{Catalyst Preparation}

\subsubsection{Preparation of $\left[\mathrm{C}_{3} \mathrm{SO}_{3} \mathrm{Hnhm}\right]_{3} \mathrm{PW}_{12} \mathrm{O}_{40}$}

1, 3-propane sultone $(6.107 \mathrm{~g}, 0.05 \mathrm{~mol})$ was dissolved in $50 \mathrm{~mL}$ acetone, which was put in a $100 \mathrm{~mL}$ three-necked flask in ice-bath. Then, morpholine $(4.356 \mathrm{~g}, 0.05 \mathrm{~mol})$ was added into the aforementioned solution drop by drop by dropping funnel. After $1 \mathrm{~h}$ of reaction, the resulting white solid was washed three times with $30 \mathrm{~mL}$ acetone and dried under vacuum at $80{ }^{\circ} \mathrm{C}$ for $24 \mathrm{~h}$ to obtain the intermediate 1-(3-sulfopropyl) morpholine. A mixture of 1-(3-sulfopropyl) morpholine (6.240 g, 0.03 $\mathrm{mol})$ synthesized in the former experimental steps, phosphotungstic acid $(28.801 \mathrm{~g}, 0.01 \mathrm{~mol})$ and deionized water $(50 \mathrm{~mL})$ was stirred for $24 \mathrm{~h}$ at room temperature. The aforementioned resulting solution was dehydrated by vacuum drying for $24 \mathrm{~h}$ at $80^{\circ} \mathrm{C}$. Then, a gray-green solid was obtained and it was named $\left[\mathrm{C}_{3} \mathrm{SO}_{3} \mathrm{Hnhm}\right]_{3} \mathrm{PW}_{12} \mathrm{O}_{40}(\mathrm{CSPW})$.

\subsubsection{Preparation of MIL-101}

Detailed preparation process of the MIL-101 was listed as follows: $\mathrm{Cr}\left(\mathrm{NO}_{3}\right)_{3} \cdot 3 \mathrm{H}_{2} \mathrm{O}(4.000 \mathrm{~g})$ and terephthalic acid $(1.660 \mathrm{~g})$ were dissolved in deionized water $(48 \mathrm{~mL})$ under the ultrasonic treatment for $10 \mathrm{~min}$. And then, hydrofluoric acid $(0.4 \mathrm{~mL})$ was added into the aforementioned solution and dispersed by ultrasonic treatment for $5 \mathrm{~min}$. The obtained mixture was sealed in a Teflon-lined autoclave and kept in an oven for $8 \mathrm{~h}$ at $220^{\circ} \mathrm{C}$. Then, the aforesaid obtained green precipitates was isolated by centrifugation after cooling down to room temperature, and the obtained solid product was washed with DMF and ethanol to remove unreacted terephthalic acid and water on the surface of the composite. And finally the green powder (the original MIL-101) was obtained by drying in oven overnight at $80^{\circ} \mathrm{C}$. 


\subsubsection{Preparation of X\%CSPW@MIL-101}

Encapsulation of $\left[\mathrm{C}_{3} \mathrm{SO}_{3} \mathrm{Hnhm}\right]_{3} \mathrm{PW}_{12} \mathrm{O}_{40}$ into the MIL-101 (X\%CSPW@ MIL-101) was performed by in-situ assembly, which the synthesis process was similar with procedure of the former original MIL-101 (section 2.2.2). The detailed preparation process as follows: $\mathrm{Cr}\left(\mathrm{NO}_{3}\right)_{3} \cdot 3 \mathrm{H}_{2} \mathrm{O}(4.000 \mathrm{~g})$ and terephthalic acid $(1.660 \mathrm{~g})$ were dissolved in $48 \mathrm{~mL}$ deionized water by using ultrasound for $10 \mathrm{~min}$. Then a given amount of $\left[\mathrm{C}_{3} \mathrm{SO}_{3} \mathrm{Hnhm}\right]_{3} \mathrm{PW}_{12} \mathrm{O}_{40}$ and hydrofluoric acid $(0.4 \mathrm{~mL})$ was successively added into the solution and dispersed by ultrasonic treatment for $5 \mathrm{~min}$. The obtained mixture was sealed in a Teflon-lined autoclave and kept in an oven for $8 \mathrm{~h}$ at $220^{\circ} \mathrm{C}$. The green precipitates was isolated by centrifugation after cooling down to room temperature. The obtained solid was washed with DMF and ethanol to remove unreacted terephthalic acid and crystal water on the surface of the composite. And finally the green powder was obtained by drying in oven overnight at $80^{\circ} \mathrm{C}$. When the addition amount of $\left[\mathrm{C}_{3} \mathrm{SO}_{3} \mathrm{Hnhm}\right]_{3} \mathrm{PW}_{12} \mathrm{O}_{40}$ was $1.000 \mathrm{~g}, 2.000 \mathrm{~g}, 3.000 \mathrm{~g}$ and $4.000 \mathrm{~g}$, which was corresponding to the $\left[\mathrm{C}_{3} \mathrm{SO}_{3} \mathrm{Hnhm}\right]_{3} \mathrm{PW}_{12} \mathrm{O}_{40}$ weight content was $7 \%, 16 \%, 31 \%$ and $48 \%$ in the final product, respectively (the aforementioned amount of $\left[\mathrm{C}_{3} \mathrm{SO}_{3} \mathrm{Hnhm}\right]_{3} \mathrm{PW}_{12} \mathrm{O}_{40}$ was evaluated by using X-ray Fluorescence (XRF) spectroscopy.) The resulting catalysts were denoted as 7\%CSPW@MIL-101, 16\%CSPW@MIL-101, 31\%CSPW@MIL-101 and 48\%CSPW @MIL-101 in this research.

\subsection{Characterization}

Crystallinity of catalyst powder was characterized by a D/max $2500 \mathrm{v} / \mathrm{pc}$ (Rigaku, Japan) X-ray diffractometer in the range of $5^{\circ} \sim 80^{\circ}$ at the speed of $5^{\circ} \cdot \mathrm{min}^{-1}$. The chemical structures of $\left[\mathrm{C}_{3} \mathrm{SO}_{3} \mathrm{Hnhm}\right]_{3} \mathrm{PW}_{12} \mathrm{O}_{40}, \mathrm{MIL}-101$ and X\%CSPW@MIL-101 were analyzed by FT-IR spectrometer (Perkin-Elmer, USA). Scanning electron microscope (SEM) images of the catalyst samples were taken with JEM-2100F( Hitachi, Japan) by gold coating. Thermal stability of the catalyst samples was recorded by Q600 (TA Instrument company, USA) at a heating rate of $10^{\circ} \mathrm{C} \mathrm{min}^{-1}$ and $\mathrm{N}_{2}$ atmospheres. Nitrogen sorption analysis was carried out at $-196^{\circ} \mathrm{C}$ using ASIQM0002-3 automatic physisorption-chemisorption apparatus (American quantachrome instrument company, USA). The samples were degassed under vacuum for $3 \mathrm{~h}$ at $150^{\circ} \mathrm{C}$. The BET (4-point evaluation) and BJH (performed on the desorption branch) equations were applied to determine the BET surface area and the pore size distribution, respectively. The element content of the samples were analyzed using a S8 Tiger Xray fluorescence spectrometer (XRF) (Bruker, Germany). The oxidation reaction solution is analyzed by gas chromatography-mass spectrometer (GC-MS) (7890A/5975C, HP-5MS column)(Agilent, USA ).

${ }^{1} \mathrm{H}-\mathrm{NMR}$ spectra of samples were measured with a AV ANCE III 400 spectrometer (Bruker, Germany) at ambient temperature in $\mathrm{D}_{2} \mathrm{O}$ and DMSO- $\mathrm{D}_{6}$ using TMS as internal reference. ( $\left[\mathrm{C}_{3} \mathrm{SO}_{3} \mathrm{Hnhm}\right]_{3} \mathrm{PW}_{12} \mathrm{O}_{40}:{ }^{1} \mathrm{H} \mathrm{NMR}\left(400 \mathrm{MHz}, \mathrm{D}_{2} \mathrm{O}\right), \delta: 2.31 \sim 2.38(\mathrm{~m}$, $\left.2 \mathrm{H}, \mathrm{NCH}_{2} \mathrm{CH}_{2} \mathrm{CH}_{2} \mathrm{SO}_{3}^{-}\right), 2.89-2.31\left(\mathrm{~m}, 2 \mathrm{H}, \mathrm{NCH}_{2} \mathrm{CH}_{2} \mathrm{CH}_{2} \mathrm{SO}_{3}{ }^{-}\right), 3.14 \sim 3.26\left(\mathrm{~s}, 2 \mathrm{H}, \mathrm{CH}_{2} \mathrm{NCH}_{2}\right), 3.15 \sim 3.27(\mathrm{~m}, 4 \mathrm{H}$, $\left.\mathrm{NCH}_{2} \mathrm{CH}_{2} \mathrm{CH}_{2} \mathrm{SO}_{3}^{-}\right)$, 3.95 4.03 (m, 4H, $\left.\left.\mathrm{CH}_{2} \mathrm{OCH}_{2}\right)\right)$.

Elemental analysis was tested with Vairo EL (Element system company, Germany). The temperature of oxidation furnace and reduction furnace was $950^{\circ} \mathrm{C}$ and $500^{\circ} \mathrm{C}$, respectively. $\left(\left[\mathrm{C}_{3} \mathrm{SO}_{3} \mathrm{Hnhm}\right]_{3} \mathrm{PW}_{12} \mathrm{O}_{40}: \mathrm{w}(\mathrm{C})=7.18 \%, w(\mathrm{H})=1.37 \%, w(\mathrm{~N})=1.19 \%\right.$ [Theoretical value: $w(C)=7.19 \%, w(H)=1.38 \%, w(N)=1.20 \%])$.

\subsection{Catalytic reaction}

Catalytic reaction of cyclohexene was carried out in a $50 \mathrm{~mL}$ three-necked flask equipped with a water condenser, a thermometer, a magnetic stirrer, and a constant-pressure dropping funnel. A certain amount of catalyst and $\mathrm{H}_{2} \mathrm{O}_{2}(30 \mathrm{wt} \%)$ was added into the aforementioned solution. After stirring for $10 \mathrm{~min}$, cyclohexene $(5 \mathrm{~mL})$ was added dropwise into the aforementioned solution. Then going to stir the reaction mixture until the setting reaction time, then stop heating, remove the three-necked bottle when the temperature is near to room temperature, centrifugal separation to remove the catalyst, pour out the reaction solution, The reaction solution was cooled in an ice-water bath, vacuum filtered when the crystals no 
longer was increased, and recrystallized after washing with cold water. The pure white crystals were obtained after suction filtration and drying in oven for $24 \mathrm{~h}$ at $80^{\circ} \mathrm{C}$. The remaining solution was analyzed by GC-MS. The method for calculating the yield of $A A$ is the ratio of the experimentally obtained $A A$ crystal mass to the theoretical AA crystal mass.

\section{Results And Discussion}

\subsection{Catalyst characterization}

XRD patterns of a series of materials synthesized in this paper were shown in Fig. 1. As showed in Fig. 1, the diffraction peaks of the synthesized MIL-101 (Fig.1c) matched with the peaks of the simulated MIL-101 (Fig. 1b), which was obtained based on the crystallography information files from the Cambridge Structure Database[22,23, 24$]$. Furthermore, in comparison to MIL-101 (Fig. 1b-1c), X\%CSPW@MIL-101 (Fig. 1d-1g) were also retained the Bragg characteristic peaks at the same positions ( $2 \theta$ ranges $5-7^{\circ}$ and $8-12^{\circ}$ ), which implies that the introduction of CSPW ionic liquid did not destroy the original structure of MIL-101.

However, an obvious change in relative intensities can be seen at 5-7 $7^{\circ}$ that indicates the encapsulation of CSPW ionic liquid within the pores of MIL-101 changed the crystal structure, which was due to the loading of CSPW ionic liquid. Such similar phenomenon also has been found in those heteropolyacid ionic liquid@MIL-101[25]. This may be due to the strong interaction between the heteropolyacid ionic liquid and MIL-101, which was distorted the MIL-101 lattice in the composite. In addition, no obvious characteristic peaks of heteropolyacid ionic liquid were found in the composite material, which can also indicate that the ionic liquid phosphotungstate is relatively uniformly distributed in the composite material, and there is no aggregation to form large grains, which cannot be detected by XRD. Moreover, there is no difference in diffraction peaks between the regenerated catalyst after six consecutive cycles (Fig. 1(a)) and the original synthesized catalyst (Fig. 1(f)). The result confirmed that the structure of the original catalyst remained unchanged after 6 time catalytic cycles.

FT-IR spectra of the different materials were shown in Fig. 2. The bands at $1402 \mathrm{~cm}^{-1}$ were attributed to $v(\mathrm{C}-0)$ stretching vibrations, and the characteristic absorption of $v(\mathrm{C}=\mathrm{C})$ vibrations $\left(1512\right.$ and $\left.1626 \mathrm{~cm}^{-1}\right)$ and $v(\mathrm{C}-\mathrm{H})$ vibrations $\left(748 \mathrm{~cm}^{-1}\right)$ (Fig. 2(2))were observed corresponding to aromatic groups[26]. The vibrational band at $1017 \mathrm{~cm}^{-1}$ were also observed, which may be attributed to $\mathrm{Cr}-\mathrm{O}$ bond formed by $\mathrm{O}=\mathrm{C}-\mathrm{O}$ (terephthalic acid) and $\mathrm{Cr}^{3+}\left[{ }^{27}\right]$.

For the X\%CSPW@MIL-101 samples (Fig. 2(2) c-f), four additional bands corresponding to phosphotungstate at 1092, 969, 890 , and $815 \mathrm{~cm}^{-1}$ were assigned to $\mathrm{v}_{\mathrm{as}}\left(\mathrm{P}-\mathrm{O}_{\mathrm{a}}\right), \mathrm{v}_{\mathrm{as}}\left(\mathrm{W}-\mathrm{O}_{\mathrm{d}}\right), \mathrm{v}_{\mathrm{as}}\left(\mathrm{W}-\mathrm{O}_{\mathrm{b}}-\mathrm{W}\right)$, and $\mathrm{v}_{\mathrm{as}}\left(\mathrm{W}-\mathrm{O}_{\mathrm{c}}-\mathrm{W}\right)$ vibrations, respectively[ $\left.{ }^{28}\right]$. Compared with the spectrum of $\left[\mathrm{C}_{3} \mathrm{SO}_{3} \mathrm{Hnhm}\right]_{3} \mathrm{PW}_{12} \mathrm{O}_{40}\left(1077,983,897\right.$ and $\left.815 \mathrm{~cm}^{-1}\right)$, the vibrational frequencies were observed with a slight red shift, which was due to the interactions between CSPW and MIL-101, which indicating that the CSPW has confined in the cages of MIL-101. The aforementioned results are consistent with the literature[ $\left.{ }^{29}\right]$. Moreover, the typical characteristic bands of the sulfonic acid group were also found at 1260 and $1042 \mathrm{~cm}^{-1}\left[{ }^{30}\right]$, which were assigned to the $v(\mathrm{~S}=0)$ asymmetric and symmetric stretching vibrations. Meanwhile, the characteristic peaks (Fig. 2(2)a) of the regenerated catalyst after six consecutive catalytic cycles are in agreement with those of the fresh catalyst (Fig. 2(2)e).

Fig. 3 shows the characteristic result of $\mathrm{N}_{2}$ adsorption-desorption isotherms and pore size distribution of original MIL-101 and X\%CSPW@MIL-101. According to the International Union for Pure and Applied Chemistry \IUPAC『classification, Type-I adsorption was observed with all the samples. This result indicates that the microporous nature was maintained even after introduction of the CSPW into the MOF structure. It also can be seen from Fig. 3 that when $P / P_{0}$ is between 0 and 0.23 , the adsorption capacity increases sharply in two stages with the increase of pressure, which was indicated that there are two kind pore structures of different sizes in MIL-101. There is a sharp rise in $P / P_{0}$ between 0.9 and 1.0, which may be caused by the increase of the pore sizes of MIL-101 due to the addition of CSPW. And when the P/P $\mathrm{P}_{0}$ is between 0.23 and 0.91 , the adsorption capacity increases slowly and reaches the saturation. 
Table 1 Pore sizes distribution of MIL-101 and X\%CSPW@MIL-101

\begin{tabular}{|llllll|}
\hline Sample & $\begin{array}{l}\text { Size of larger } \\
\text { mesocage } \\
(\mathrm{nm})\end{array}$ & $\begin{array}{l}\text { Size of small } \\
\text { mesocage } \\
(\mathrm{nm})\end{array}$ & $\begin{array}{l}\text { Size of larger } \\
\text { window } \\
(\mathrm{nm})\end{array}$ & $\begin{array}{l}\text { Size of small } \\
\text { window } \\
(\mathrm{nm})\end{array}$ & $\begin{array}{l}\text { Particle size } \\
(\mathrm{um})\end{array}$ \\
\hline MIL-101 & 3.1913 & 2.4361 & 1.57 & 0.8227 & $0.35 \sim 0.50$ \\
\hline $\begin{array}{l}7 \% \text { CSPW@MIL- } \\
101\end{array}$ & 3.0664 & 2.3045 & 1.635 & 0.8505 & $0.51-0.87$ \\
\hline $\begin{array}{l}16 \% \text { CSPW@MIL- } \\
101\end{array}$ & 2.9416 & 2.2699 & 1.635 & 0.8641 & $0.36 \sim 0.94$ \\
\hline $\begin{array}{l}31 \% \text { CSPW@MIL- } \\
101\end{array}$ & 2.9416 & 2.2353 & 1.6293 & 0.8294 & $\begin{array}{l}\text { Agglomerated } \\
\text { particles }\end{array}$ \\
\hline $\begin{array}{l}48 \% \text { CSPW@MIL- } \\
101\end{array}$ & 2.7550 & 2.0277 & 1.6293 & 0.8294 & $\begin{array}{l}\text { Agglomerated } \\
\text { particles }\end{array}$ \\
\hline
\end{tabular}

Meanwhile, pore sizes distribution (PSD) of the catalyst synthesized in this paper was calculated from density function theory (DFT) method and the result was showed in Fig. 3 and Table. 1, which was illustrated that there were two kind mesocages structure (3.2 and $2.4 \mathrm{~nm}$ ), and corresponding to the two windows (1.6 and $0.8 \mathrm{~nm}$ ) in MIL-101. Moreover, the aforementioned result demonstrated that the $\left[\mathrm{C}_{3} \mathrm{SO}_{3} \mathrm{Hnhm}\right]_{3} \mathrm{PW}_{12} \mathrm{O}_{40}$ (molecular diameter is $2.1 \mathrm{~nm}$ ) will confined into the cages structure of the catalyst. For this reason, the loss of active components can be significantly reduced, and that was because the uniform microporous window of MIL-101 is a natural "molecular sieve", that is, cyclohexene $(0.42 \mathrm{~nm})$ molecules smaller than its window can enter the pores to reach the active center, while molecules larger than its window cannot enter. On the other hand, the X\%CSPW@MIL-101 micron-level (Table 1) hollow octahedral structure can be easily recovered by centrifugation. Therefore, the catalyst synthesized in this research can be used in size-selective catalysis, which will display the excellent catalytic activity, substrate molecular size selectivity and recycling stability. Furthermore, from the Fig. 3 and Table 1, it was also seen that the pore size distribution peak of the X\%CSPW@MIL-101 was shifted to the left compared with the peak of original MIL-101, and the amplitude of the peak shift to the left was greater with the loading amount of CSPW was increased, which was due to the introduction of CSPW[28].

Table 2 presents the physicochemical properties of the sample. The BET surface areas of MIL-101 synthesized in this research were in agreement with those of the samples as reported in other literature[ ${ }^{31]}$. Compared with the pristine MIL-101, the X\%CSPW@MIL-101 samples demonstrated a significant decrease trends in both pore volume (from $1.301 \mathrm{~cm}^{3} \mathrm{~g}^{-1}$ to $0.541 \mathrm{~cm}^{3} \mathrm{~g}^{-1}$ ) and surface area (from $2535 \mathrm{~m}^{2} \mathrm{~g}^{-1}$ to $1208 \mathrm{~m}^{2} \mathrm{~g}^{-1}$ ), which was due to the encapsulation of CSPW into the cages of the MIL-101.

Table 2 Surface area measurements for MIL-101 and X\%CSPW@MIL-101 


\begin{tabular}{|lllll|}
\hline Sample & $\begin{array}{l}\text { BET surface area } \\
\left(\mathrm{m}^{2} \mathrm{~g}^{-1}\right)\end{array}$ & $\begin{array}{l}\text { DFT method Pore volume } \\
\left(\mathrm{cm}^{3} \mathrm{~g}^{-1}\right)\end{array}$ & $\begin{array}{l}\text { DFT pore diameter } \\
(\mathrm{nm})\end{array}$ & $\begin{array}{l}\text { Average pore } \\
\text { Diameter }(\mathrm{nm})\end{array}$ \\
\hline MIL-101 & 2535 & 1.301 & 2.504 & 2.284 \\
\hline $\begin{array}{l}7 \% \text { CSPW@MIL- } \\
101\end{array}$ & 2478 & 1.230 & 2.380 & 2.191 \\
\hline $\begin{array}{l}16 \% \text { CSPW@MIL- } \\
101\end{array}$ & 2372 & 1.150 & 2.266 & 2.100 \\
\hline $\begin{array}{l}31 \% \text { CSPW@MIL- } \\
101\end{array}$ & 1896 & 0.877 & 2.941 & 1.995 \\
\hline $\begin{array}{l}48 \% \text { CSPW@MIL- } \\
101\end{array}$ & 1208 & 0.541 & 2.703 & 1.914 \\
\hline
\end{tabular}

Combined with the FT-IR measurement, we concluded that CSPW was successfully encapsulated into the micropores of MIL-101 rather than outside the surfaces, which were without any destruction for the framework structure of the MIL-101.

SEM and SEM-EDS images of the catalyst sample synthesized in this research were illustrated in Fig. 4. As can be seen from Fig. 4 (a), dominant crystal shapes of MIL-101 is octahedral, which ranges in size between 0.35 and 0.50 um. However, the dominant crystal shape of the X\%CSPW@MIL-101(Fig. 4(b), (c), (d) and (e)) are a monolithic, which is made up of different sizes of octahedra ranging in size from 0.5 to $1.0 \mathrm{um}$. This emergence of the aforementioned experimental phenomenon may be due to the fact that the nitrogen ion on the morpholine ring of the cation in the ionic liquid phosphotungstate is an electron-rich group, which can form a coordination compound with the $\mathrm{Cr}^{3+}$ on MIL-101, accelerating the formation of the secondary structural unit of MIL-101, aggregating, and eventually forming clustered particles in irregular fragments. This is consistent with the research results of $\mathrm{Hu}[28]$.

Fig. $4(\mathrm{f}),(\mathrm{g})$ and $(\mathrm{h})$ provide the SEM-EDS images of 31\%CSPW@MIL-101 composite material. It can be seen that there are seven elements of W, P, S, N, C, O and Cr on the surface of the 31\%CSPW@MIL-101 catalyst. Among them, W, P, S and N come from the ionic liquid phosphotungstate, and $\mathrm{C}, \mathrm{O}$ and $\mathrm{Cr}$, come from MIL-101. It can be seen from the element distribution diagram that the ion was uniform distribution of the phosphotungstate on the surface of the composite material and also shows that the CSPW can be well dispersed in the composite material. And this aforementioned result is consistent with the XRD analysis result shown in Fig. 1.

\subsection{Catalytic performance}

Catalytic performance of different catalysts synthesized in this research were showed in the Fig. 5, which was obtained under the experimental conditions was that the catalyst dose was $1.0 \mathrm{~g}$, cyclohexene was $5 \mathrm{~mL}, \mathrm{H}_{2} \mathrm{O}_{2}(30 \mathrm{wt} \%)$ was $22 \mathrm{~mL}$, reaction time was $10 \mathrm{~h}$ and reaction temperature was $90^{\circ} \mathrm{C}$.

From Fig. 5, it can see that the typical MIL-101 itself have only a little catalytic activity, and its AA yield only was $1.6 \%$. The reason may be that the backbone node of MIL-101 directly catalyzes the oxidation of olefins[ ${ }^{32}$. And the X\%CSPW@MIL101 catalyst showed remarkable catalytic activity, which was due to the CSPW in the composite material plays a key role in this catalytic oxidation reaction. This was because in the oxidation reaction process, $\mathrm{H}_{2} \mathrm{O}_{2}$ was used as an oxidant to oxidize the phosphotungstate to form tungsten peroxo species, and the tungsten peroxo species can catalyze the epoxidation of cyclohexene as real active oxidants $\left[14,{ }^{33}\right]$. Meanwhile, from Fig. 5 it also can be observed that with the increase of the encapsulating amount of CSPW, the yield of AA gradually increased. When the amount of encapsulating of CSPW reached $31 \%$, the composite catalyst showed the largest catalytic activity, and the yield of AA reached $81.2 \%$, which was almost close to the catalytic activity of original $\left[\mathrm{C}_{3} \mathrm{SO}_{3} \mathrm{Hnhm}\right]_{3} \mathrm{PW}_{12} \mathrm{O}_{40}$ (AA yield is $85.3 \%$, under the same amount of 
catalyst), which shows that the strategy of packaging CSPW with MIL-101 in this experiment is feasible. This also shows that the CSPW plays a leading role in the oxidation reaction. It is worth noting that when the encapsulation content of CSPW exceeds $31 \%$, the catalytic activity for cyclohexene decreases, because the excess loading of CSPW will result in the smaller size of mesocage and surface, which affects the activity of the composite catalyst. Moreover the excess loading of CSPW will leach from the MOF framework, and the activity of the composite catalyst will also affect.

Through the investigation of the reaction process conditions, we found that the reaction temperature and reaction time were important factors affecting the catalytic reaction, which the aforementioned factors investigated the results were shown in Fig. 6. When the reaction temperature was lower than $75^{\circ} \mathrm{C}$, the reaction hardly produced $\mathrm{AA}$. When the reaction temperature of the reaction system up to $80^{\circ} \mathrm{C}$, the reflux began. At this time, a certain amount of $\mathrm{AA}$ was produced, but the yield of AA was still lower than $10 \%$. When the reaction temperature was further increased to $85^{\circ} \mathrm{C}$, the yield of $A A$ had been significantly increased, which was attributed to the relatively high temperature favoring the improvement of the reaction rate. Then the reaction temperature rose to $95^{\circ} \mathrm{C}$ and the yield of $\mathrm{AA}$ gradually reached saturation.

It is worth noting that when the reaction temperature increased from $95^{\circ} \mathrm{C}$ to $100{ }^{\circ} \mathrm{C}$, although the yield of $\mathrm{AA}$ was still increasing, but it was only increased by $0.9 \%$. From a practical point of view, we hope to obtain a high-purity AA, but at the same time, the increase in temperature also causes excessive invalid decomposition of $\mathrm{H}_{2} \mathrm{O}_{2}$. We investigated that the decomposition amount of $\mathrm{H}_{2} \mathrm{O}_{2}$ reached $10 \%$ at $100^{\circ} \mathrm{C}$, so we chose the optimal reaction temperature is $95^{\circ} \mathrm{C}$. Meanwhile, we also investigated the effect of reaction time on the catalytic reaction at different temperature. In general, the yield of AA increases gradually with reaction time, and the reaction time to reach the same yield varies at different temperatures. When the reaction was performed at the optimal temperature of $95^{\circ} \mathrm{C}$, the reaction proceeded to $10 \mathrm{~h}$, and the yield of $A A$ was close to the saturation, and further to prolong the reaction time, the yield of AA did not change significantly, so the optimal reaction time was determined to be $10 \mathrm{~h}$.

In this paper, the effect of the catalyst dose on the yield of AA was carefully investigated (Fig. 7). From Fig. 7, it was seen that the catalyst dose mainly affects the reflux time of the reaction and then the yield of AA. With the increase of the catalyst dose, the yield of AA first increased to the highest, and then gradually decreased. When the catalyst dose was 1.0 $\mathrm{g} / 5 \mathrm{~mL}$ cyclohexene, an appropriate number of catalyst activity centers can effectively catalyze the reaction and maximize the AA yield. It is worth noting that the catalytic activity decreases when the catalyst dose exceeds $1 \mathrm{~g} / 5 \mathrm{~mL}$ cyclohexene, which the reason should be that the relative excess of active sites will aggravate side reactions and generate other carboxylic acids and epoxides, which will affect the yield of AA.

On the other hand, in this experiment, the effect of molar ratio of $\mathrm{H}_{2} \mathrm{O}_{2}$ to cyclohexene on the yield of AA was also carefully investigated (Fig. 8). From Fig. 8, it was seen that the highest yield of AA was not 4:1, which was corresponding to the theoretical molar ratio of $\mathrm{H}_{2} \mathrm{O}_{2}$ to cyclohexene in the reaction of clean catalytically catalyzed oxidation of cyclohexene to AA. These aforementioned result from some of the inefficient decomposition of $\mathrm{H}_{2} \mathrm{O}_{2}$ reduced its utilization rate under the progress of the reaction. Meanwhile, some $\mathrm{H}_{2} \mathrm{O}_{2}$ was also used as an oxidant to oxidize the phosphotungstate to form tungsten peroxo species. Since the catalytic reaction is a complexation catalytic reaction, the appropriate $\mathrm{H}_{2} \mathrm{O}_{2}$ concentration is conducive to the formation of the active structure of the catalyst. When the dosage ratio of $\mathrm{H}_{2} \mathrm{O}_{2}$ /cyclohexanone is 4.4, the utilization rate of $\mathrm{H}_{2} \mathrm{O}_{2}$ reaches the highest, and the maximum AA yield is also obtained. At the same time, considering the stability of the catalyst and the effect of cyclohexene transportation on the promotion of $\mathrm{H}_{2} \mathrm{O}_{2}$ decomposition, there must be a certain margin in the reaction system. So the optimal quantitative ratio of $\mathrm{H}_{2} \mathrm{O}_{2}$ /cyclohexanone was 4.4 .

\subsection{XRF}


X\%CSPW@MIL-101 catalyst synthesized in this paper was conducted by XRF analysis to determine the corresponding CSPW loading of the materials, the results were showed in the Table 3 and Fig. 9.

It can be seen that the $\mathrm{W} / \mathrm{Cr}(\mathrm{mol})$ of the catalyst increases with the amount of CSPW increases, and the average number of CSPW per cage in MIL-101 also was increased, but when the W/Cr was 0.44, the yield of AA will up to the maximum and then the CSPW content further to increase, the yield of AA was decreased. This may be because the excessive CSPW occupies the volume of the MIL-101 cage and reduces the reaction site, which is not conducive to the diffusion and oxidation of cyclohexene. Therefore, a suitable CSPW content (31\%) was more conducive to increase the yield of AA.

Table 3 XRF analysis results of X\%CSPW@MIL-101 composites materials

\begin{tabular}{|c|c|c|c|c|c|c|c|}
\hline Sample & $\mathrm{Cr}$ & W & $S$ & $P$ & 0 & CSPW $₫ w t \% \rrbracket$ & CSPW/Cage ratio \\
\hline 7\%CSPW@MIL-101ه1g》 & 29.62 & 7.46 & 1.53 & 0.07 & 19.55 & 6.65 & 1 \\
\hline 16\%CSPW@MIL-101ヌ2g】 & 30.39 & 20.07 & 1.02 & 0.21 & 21.99 & 15.75 & 1 \\
\hline 31\%CSPW@MIL-101』3g】 & 21.28 & 33.43 & 2.98 & 0.43 & 23.92 & 30.88 & 2 \\
\hline 48\%CSPW@MIL-101ه4g】 & 15.17 & 48.59 & 1.27 & 0.59 & 22.34 & 47.55 & 3 \\
\hline Regenerated (6 cycles) & 21.35 & 33.29 & 2.94 & 0.42 & 23.92 & 30.61 & 2 \\
\hline 31\%CSPW@MIL-101®3g囚 & & & & & & & \\
\hline
\end{tabular}

\subsection{Comparison of catalytic performance}

Catalytic performance of the 31\%CSPW@MIL-101 was compared with the other reported solid catalysts and the corresponding results are summarized in Table 4. The optimal yield of AA in this present work was $81.2 \%$, which was much higher than most of the reported values using tungstate catalysts. Although $85 \%$ AA yield was achieved over the $\mathrm{H}_{2} \mathrm{WO}_{4}$ as catalyst, but the used solvent was much more than this work. In addition, other catalysts require a certain amount of solvent, such as ionic liquid, acetonitrile, etc., in order to achieve a certain yield of AA, thus they will increase solvent cost and difficulty of the separation process. The $\mathrm{K}_{3} \mathrm{PW}_{12} \mathrm{O}_{40}$ even needs $24 \mathrm{~h}$ of reaction time to reach a yield of $77 \%$. Accordingly, the 31\%CSPW@MIL-101 synthesized in this research shows a better comprehensive catalytic performance as compared with other heterogeneous solid catalytic systems under the investigated reaction conditions.

Table 4 Comparison of catalytic performance between X\%CSPW@MIL-101 and reported solid catalysts 


\begin{tabular}{|c|c|c|c|c|c|c|c|}
\hline \multirow[t]{2}{*}{ Catalysts } & \multicolumn{5}{|c|}{ Experimental conditions } & \multirow{2}{*}{$\begin{array}{l}\text { Yield } \\
\text { of } \\
\text { AA } \\
(\%)\end{array}$} & \multirow[t]{2}{*}{ References } \\
\hline & $\begin{array}{l}\text { Catalyst } \\
\text { amount } \\
\text { (g) }\end{array}$ & $\begin{array}{l}\text { Cyclohexene } \\
\text { amount } \\
\text { (mmol) }\end{array}$ & $\begin{array}{l}\text { Solvent } \\
(\mathrm{mmol})\end{array}$ & $\begin{array}{l}\text { Temperature } \\
\left({ }^{\circ} \mathrm{C}\right)\end{array}$ & $\begin{array}{l}\text { Time } \\
\text { (h) }\end{array}$ & & \\
\hline 31\%CSPW@MIL-101 & 1.0 & 50 & none & 90 & 10 & 81.2 & This work \\
\hline $\mathrm{K}_{3} \mathrm{PW}_{12} \mathrm{O}_{40}$ & \multicolumn{2}{|c|}{$\begin{array}{l}9.5 \% \mathrm{~m} / \mathrm{m} \\
\text { (catalyst/cyclohexene) }\end{array}$} & 23(acetonitrile) & 75 & 24 & 77.0 & {$[11]$} \\
\hline $\mathrm{H}_{2} \mathrm{WO}_{4}$ & $0.2 \mathrm{mmol}$ & 10 & $0.2(\mathrm{IL})$ & $73-87$ & 12 & 85.0 & [13] \\
\hline$\left[\mathrm{I}^{-}-\mathrm{C}_{5} \mathrm{H}_{5} \mathrm{NC}_{16} \mathrm{H}_{33}\right]_{2} \mathrm{~W}_{2} \mathrm{O}_{3}\left(\mathrm{O}_{2}\right)_{4}$ & $0.6 \mathrm{mmol}$ & 100 & none & 90 & 20 & 78.3 & {$[34]$} \\
\hline
\end{tabular}

\subsection{Catalyst recyclability}

Fig. 10 shows the recyclability of the 31\%CSPW@MIL-101 catalysts. It can be found that the 31\%CSPW@MIL-101 catalyst still maintain the stabilizing yield of AA after six consecutive reaction cycles. When the catalyst was repeatedly used for several times, the catalytic activity of the catalyst was slightly reduced, but the yield of AA still remains above $80 \%$. This aforementioned slightly reduce in the catalytic performance may be due to the mechanical loss of the catalyst through centrifugation and filtration.

In this research, in order to determine the structural changes between the regenerated catalyst of the 31\%CSPW@MIL-101 after the 6th catalytic circles and the fresh catalyst of 31\%CSPW@MIL-101, a series of comparative characterizations were performed through FT-IR spectroscopy, XRF, and XRD. As showed in Fig. 2, the difference between the FT-IR spectra of the fresh (Fig. 2(2)(e)) and the regenerated catalyst (Fig. 2(2)(a)) is insignificant. The characteristic absorption peak of the sulfonic acid group (1260 and $1042 \mathrm{~cm}^{-1}$ ) and the characteristic absorption peak of Keggin heteropolyacid anion (1092, 969,890 , and $815 \mathrm{~cm}^{-1}$ ) were all can be observed clearly in the spectra of two samples.

It can be seen from Fig. 1(a) and Fig. 1(f) that there is basically no change in XRD of the catalyst that has been repeatedly used for 6 times. Additionally, the W content of the 31\%CSPW@MIL-101(Table 3), determined by XRF, the W content has basically not decreased, and demonstrates that the CSPW was restricted into the large and small cages structure of MIL101. This was in agreement with the molecular size of ionic liquid phosphotungstate and the structure and size data of MIL101 large and small cages.

\subsection{Plausible Reaction Mechanism}

$\mathrm{N}_{2}$ physical adsorption and desorption characterization results show that the pore size of X\%CSPW@MIL-101 catalyst was 2.0 and $3.2 \mathrm{~nm}$, and there were also two windows (1.6 and $0.8 \mathrm{~nm})$ in the aforementioned catalyst. However, the diameter of the cyclohexene molecule is $0.42 \mathrm{~nm}$, which these aforementioned sizes proved that the cyclohexene molecule can easily go through the inner structure of the catalyst. Because the catalytic reaction nanocage is relatively spacious, the reactant molecules can easily diffuse, adsorption and desorption.

According to the aforementioned structural characteristics of the X\%CSPW @MIL-101 catalyst, it can be considered that the catalyst can be used as a nanoreactor. Combining the results of catalytic activity and the summary of related literature[2] we can further speculate on the mechanism of oxidation reaction in the nanoreactor, as showed in Fig. 11. The catalytic 
reaction process in this research can be divided into 6 steps. The reaction substrate cyclohexene enters the nanocage through the window of MIL-101 through diffusion. Under the action of $\mathrm{H}_{2} \mathrm{O}_{2}$, phosphotungstic salt is catalyzed by sulfonic acid to peroxytungstate, which is the real active oxidant[14], and which the aforementioned peroxytungstate oxidizes the cyclohexene to epoxy cyclohexane. And then, upon epoxy cyclohexane ring-opening 1,2-cyclohexanedio is produced by sulfonic acid, which further triggers $3,4,5$ and 6 steps of reaction, and finally adipic acid is formed.

The reaction solution was analyzed by GC-MS (Fig. 12), it was found that there have 1, 2-Cyclohexanedio, 2-hydroxy cycolhexanone, and 2,7-0xepanedione in the solution, which are the reaction intermediate. These aforementioned production of the reaction intermediate well described the path of the cyclohexane double bond oxidation reaction (Scheme 1). The aforementioned reaction path is similar with the reaction process mentioned in the literature ${ }^{35]}$.

\section{Conclusion}

In this research, the ionic liquid phosphotungstate $\left[\mathrm{C}_{3} \mathrm{SO}_{3} \mathrm{Hnhm}\right]_{3} \mathrm{PW}_{12} \mathrm{O}_{40}$ was encapsulated in the cage of MIL-101 by the main body in-situ assembly method, and it was used as a catalyst for the oxidation of cyclohexene to production of adipic acid. The yield of adipic acid can reach the highest $81.3 \%$ under catalysis by the catalyst synthesized in this paper, which was higher than those reported for similar catalysts with solvent-free and without adding phase transfer reagents. During the in-situ generation of MIL-101 around the ionic liquid phosphotungstate, the ionic liquid phosphotungstate with suitable structural size can be encapsulated in the cage of MIL-101 and dispersed uniformly in the MIL-101 and it was difficult escaped from the window, which is beneficial for the catalyst to be reused many times without deactivation. The recycled catalytic performance of this 31\%CSPW@MIL-101 catalyst under repeat 6 times was evaluated and the result revealed that the yield in adipic acid was still higher than $80.0 \%$. On the other hand, with the increase of CSPW, the amount of MIL-101 that can encapsulate ionic liquid phosphotungstate also increases, but it is not that the more CSPW was encapsulated, the better the catalytic activity of the catalyst was obtained. Too much encapsulation of CSPW will reduce the space of the reaction site and the small space is not conducive to the diffusion of reactant molecules, so that the yield of AA decreases. Moreover, the cage of X\%CSPW@MIL-101 catalyst was regarded as a kind of nanoreactor. Because the space of the catalytic reaction nanocage was suitable, the reactant molecules can easily diffuse, adsorb and desorb, and meanwhile the CSPW active site was difficult escaped from window of MOF, which the aforementioned results indicating that in a moderately spatial heterogeneous catalytic microenvironment, the physical and chemical properties of the reaction substrate and molecular diffusion together affect the catalytic reaction performance.

\section{Declarations}

\section{Acknowledgements}

We gratefully acknowledge the Liaoning Provincial Natural Science Foundation Guidance Program Project (20180550695), Department of Education of Liaoning Province-other projects (2018CYY003), General Project of Liaoning Education Department (L2017LFW006), Liaoning Province Innovation Talent Support Program (LR2019038) of China for the financial support.

\section{References}

1 Feng J, Li M, Meng X (2019) Catal Lett 149: 1504-1512

2 Ateeq R, Mathew M, Courtie M (2016) Catal Lett 146: 788-799

3 Lesage G, Quesada Penate I, Franceschi S, Perez E, Garrigues J C, Poux M, Cognet P (2020) Catal Today 346: 40-45

4 Ravishankara A. R., Daniel J S., Portmann R W. (2009) Science 326: 123-125

Page $11 / 19$ 
5 Yoko U, Kazuhiko S (2003) Green Chem 5: 373-375

6 Sato K, Aoki M, Noyori R (1998) Science 281: 1646-1647

7 Li Z, Lin B, Zhang J, Geng F, Liu P (2006) J Mol Struct 783: 176-183

8 Li H, Zhu W, He X, Zhang Q, Pan J, Yan Y (2007) React Kinet Catal L 92: 319-327

9 Fujitani K, Mizutani T, Oida T, Kawase T (2009) J Oleo Sci 58:37-42

10 Alcaniz-Monge J, Trautwein G, Garcia-Garcia A (2014) J Mol Catal A-Chem 394: 211-216

11 Soares J C S, Gonçalves A H A, Zotin F M Z, Araújo L R R D, Gaspar A B (2018) Mol Catal 48: 223-229

12 Oguchi T, Ura T, Ishii Y, Ogawa M (1989) Chem Lett 53: 857-860

13 Wang B T, Zhang Z B, Zhang X Y, Sun S X, Wu L, Xing R (2018) Chem Pap 72: 643-649

14 Liu J, Yang G Q, Liu Y, Zhou Z, Zhang Z B, Hu X B (2021) Catal Lett 151: 147-152

15 Thompson D J, Zhang Y, Ren T (2014) J Mol Catal A-Chem 392:188-193

16 Marcì G, García-López E, Palmisano L (2009) Catal Today 144: $42-47$

17 Farhadi S, Zaidi M (2009) J Appl Catal A-Gen 354: 119-126

18 Zhang M, Zhu W, Xun S, Li H, Gu Q, Zhao Z, Wang Q (2013) Chem. Eng. J 220: 328-336

19 Mao H, Zhu K, Lu X, Zhang G, Yao C, Kong Y, Liu J (2015) J Colloid Interf Sci 446: 141-149

20 Stuart R. B, Neil R. C, Chen X M, Javier G M, Susumu K, Lars Ö, Michael O, Paik Suh M, Jan R (2013) Pure Appl Chem 85: 1715-1724

21 Côté A P, Benin A I, Ockwig N W, O’Keeffe M, Matzger A J, Yaghi O M (2005) Science 310: 1166-1170

22 Ferey G, Mellot-Draznieks C, Serre C, Millange F, Dutour J, Surble S, Margiolaki I (2005) Science 309: 2040-2041

23 Kholdeeva O A, Skobelev I Y, Ivanchikova I D, Kovalenko K A, Fedin V P, Sorokin A B (2014) Catal Today 238: 54-61

24 Canioni R, Roch-Marchal C, Secheresse F, Horcajada P, Serre C, Hardi-Dan M, Ferey G, Greneche J M, Lefebvre F, Chang J S, Hwang Y K, Lebedev $O$ I, Turner S, Van Tendeloo G (2011) J Mater Chem 21: 1226-1233

25 Wang X, Huang Y, Lin Z, Cao R (2014) Dalton T 43: 11950-11958

26 Jing R, Lu X, Zhang H, Tao P, Pan H, Hu A, Zhou D, Xia Q (2019) Chem J Chinese U 40: 755-762

27 Wang Q, Shao L, Ma Z, Xu J, Li Y, Wang C (2018) Electrochim Acta 281: 582-593

28 Hu X, Lu Y, Dai F, Liu C, Liu Y (2013) Micropor Mesopor Mat 170: 36-44

29 Wan H, Chen C, Wu Z, Que Y, Feng Y, Wang W, Wang L, Guan G, Liu X (2015)

ChemCatChem 7: 441-449

30 Jiang W, Dong L, Liu W, Guo T, Li H, Zhang M, Zhu W, Ling H (2017) RSC Adv 7: 55318-55325

31 Zhang Y M, Degirmenci V, Li C, Hensen E J M (2011) ChemSusChem 4: 59-64 
34 Zhu W S, Li H M, He X Y, Zhang Q, Shu H M, Yan Y S (2008) Catal Commun 9: 551-555

35 Lee S O, Raja R, Harris K D M, Thomas J M, Johnson B F G, Sankar G (2003) Angew. Chem. Int. Ed. 42 : 1520 - 1523

\section{Figures}

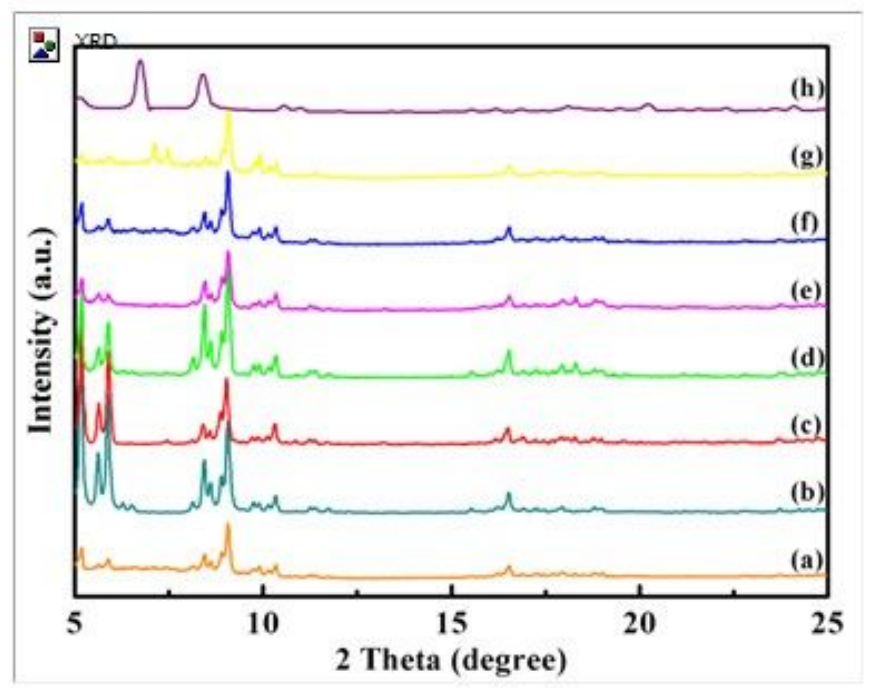

Figure 1

XRD patterns of (a) Regenerated 31\%CSPW@MIL-101, (b) Simulated MIL-101, (c) Synthesized MIL-101, (d) 7\%CSPW@MIL101, (e) 16\%CSPW@MIL-101, (f) 31\%CSPW@MIL-101, (g) 48\%CSPW@MIL-101, and (h) [C3SO3Hnhm]3PW12040 

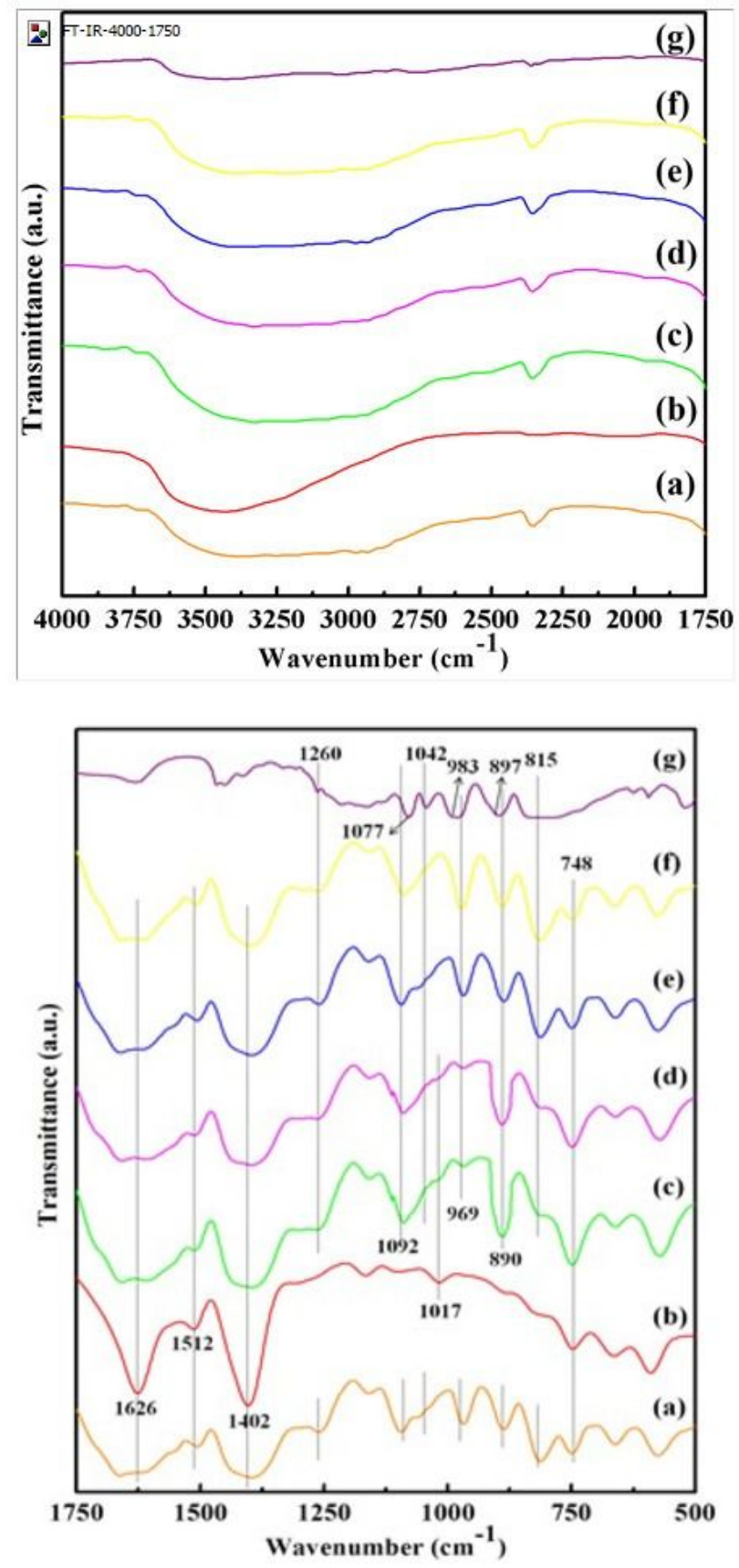

Figure 2

(1 - Top) FT-IR spectra of different materials. (a) Regenerated 31\%CSPW@MIL-101, (b) Synthesized MIL-101, (c) 7\%CSPW@MIL-101, (d) 16\%CSPW@MIL-101, (e) 31\%CSPW@MIL-101, (f) 48\%CSPW@MIL-101, and (g) [C3SO3Hnhm]3PW12040 (2 - Bottom) FT-IR spectra of different materials. (b) Regenerated 31\%CSPW@MIL-101, (b) Synthesized MIL-101, (c) 7\%CSPW@MIL-101, (d) 16\%CSPW@MIL-101, (e) 31\%CSPW@MIL-101, (f) 48\%CSPW@MIL-101, and $(\mathrm{g})[\mathrm{C} 3 \mathrm{SO} \mathrm{H} \mathrm{Hnhm}] 3 \mathrm{PW} 12 \mathrm{O} 40$ 


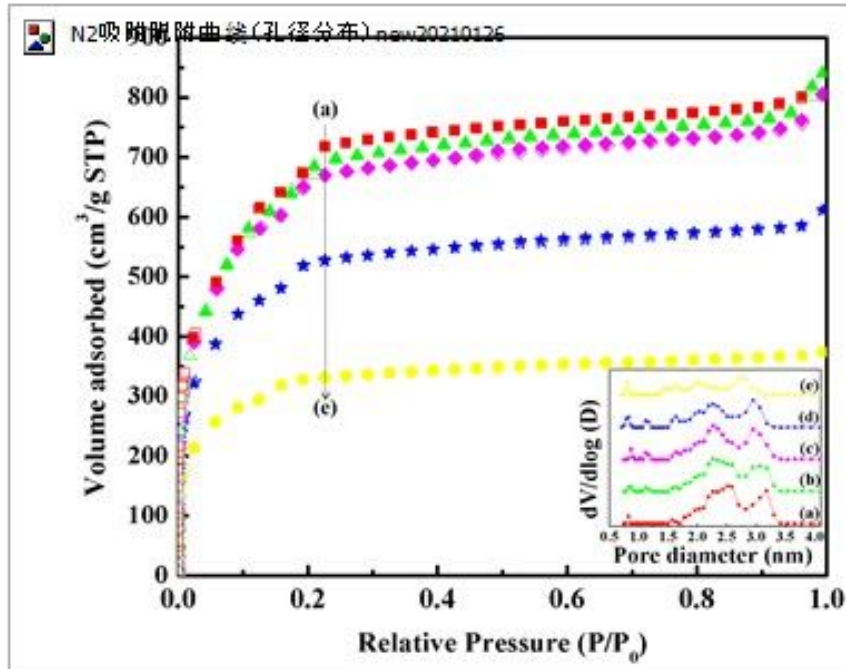

Figure 3

N2 adsorption and desorption isotherms of MIL-101 and X\%CSPW@MIL-101 (a) Synthesized MIL-101, (b) 7\%CSPW@MIL101, (c) 16\%CSPW@MIL-101, (d) 31\%CSPW@MIL-101, (e) 48\%CSPW@MIL-101
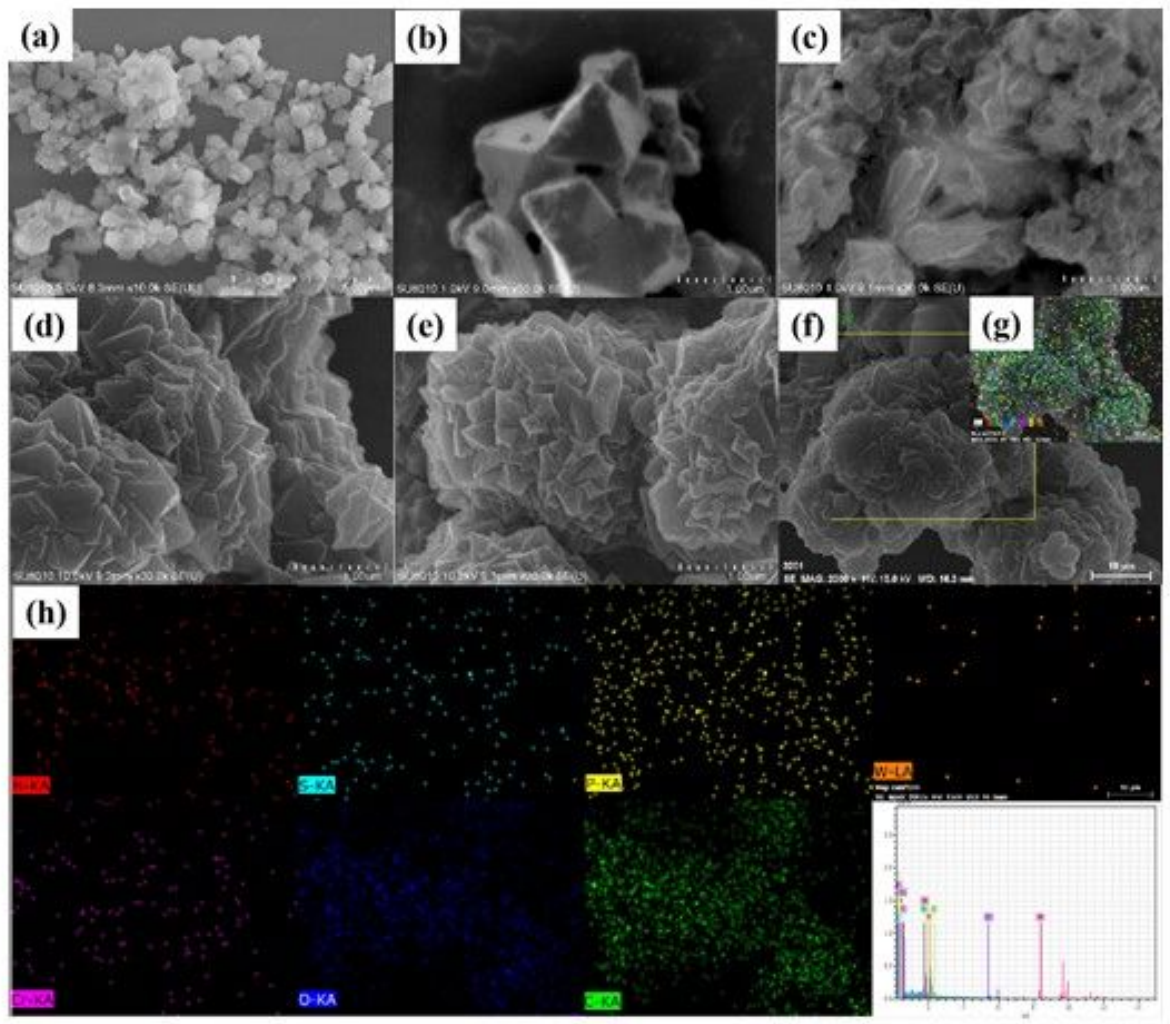

Figure 4

SEM images for (a) MIL-101, (b) 7\%CSPW@MIL-101, (c) 16\%CSPW@MIL-101, (d) 31\%CSPW@MIL-101, (e) 48\%CSPW@MIL101, (f) , (g) and (h) SEM-EDS images of 31\%CSPW@MIL-101 


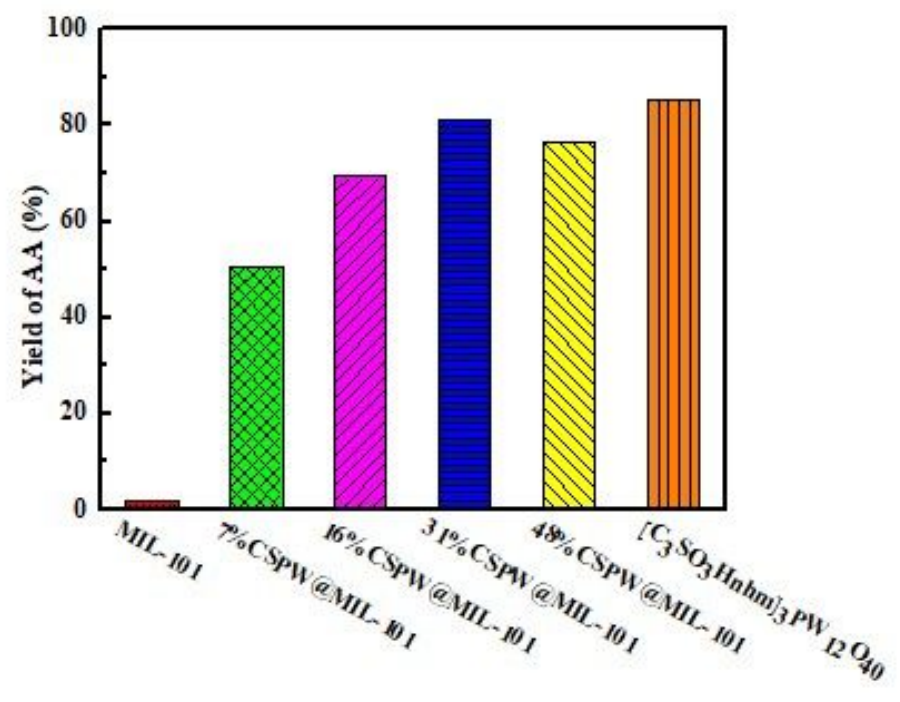

Figure 5

Catalytic performances of various catalysts for the oxidation of cyclohexene with $\mathrm{H} 2 \mathrm{O} 2$.

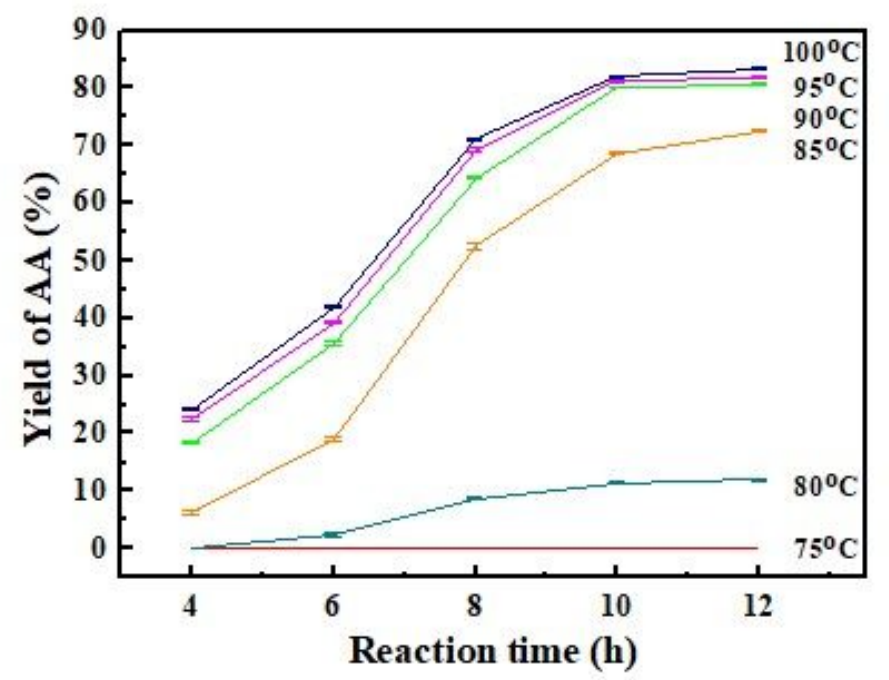

Figure 6

Effect of reaction temperature and reaction time on the yield of AA

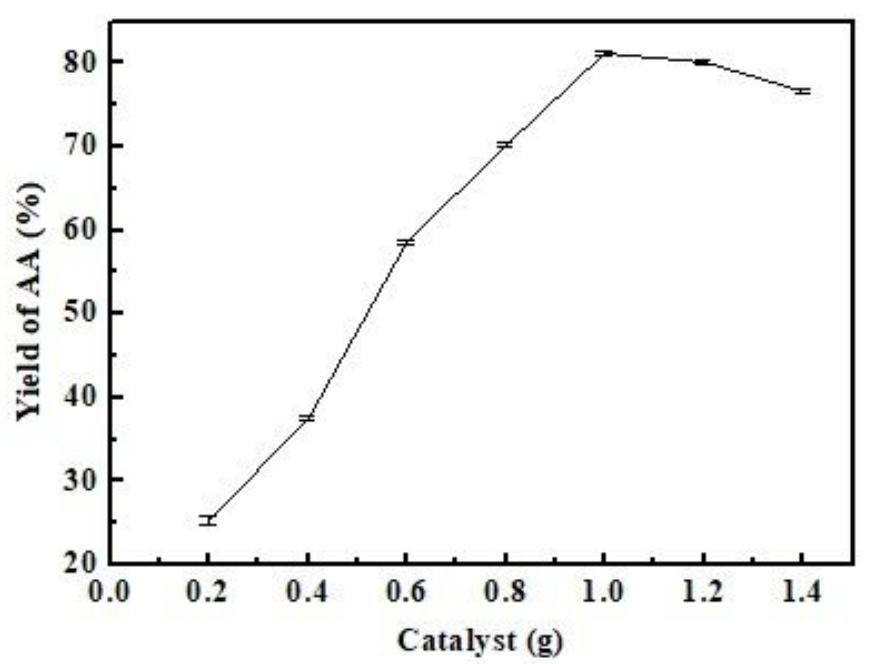


Figure 7

Effect of catalyst dose on the yield of AA

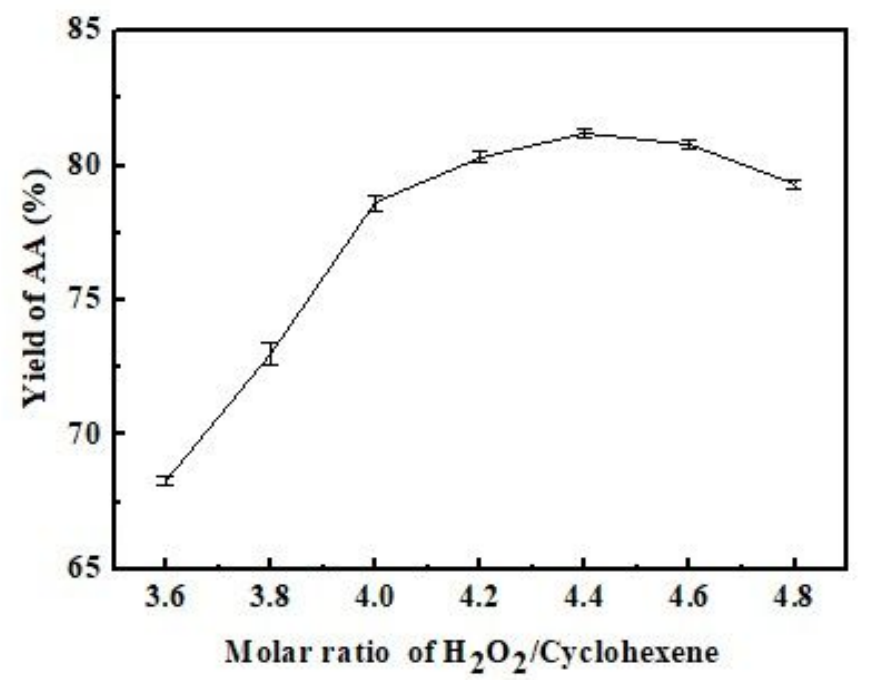

Figure 8

Effect of molar ratio of $\mathrm{H} 2 \mathrm{O} 2 /$ cyclohexene on the yield of $A A$

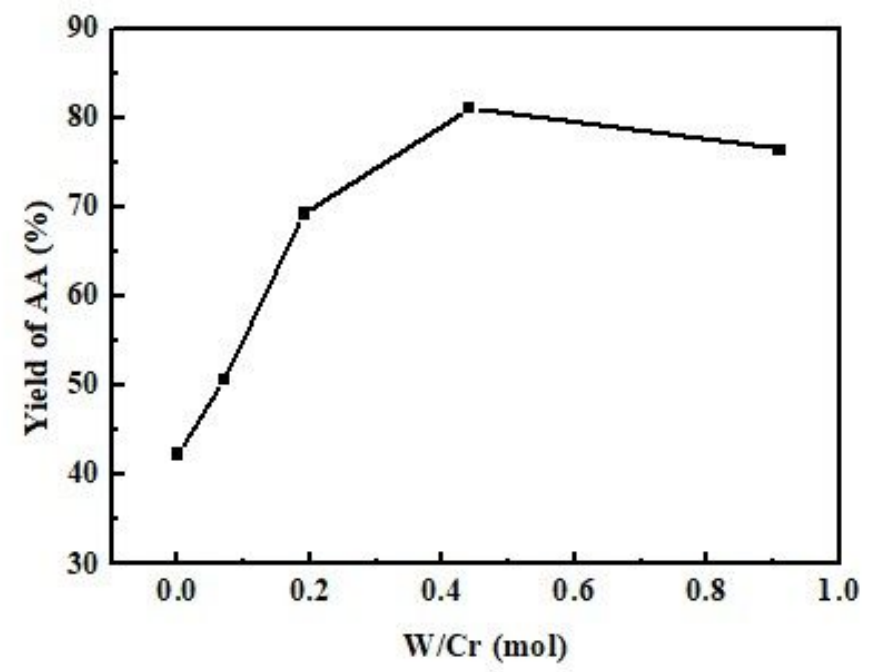

Figure 9

Effect of W/Cr(mol) of X\%CSPW@MIL-101 to yield of AA 


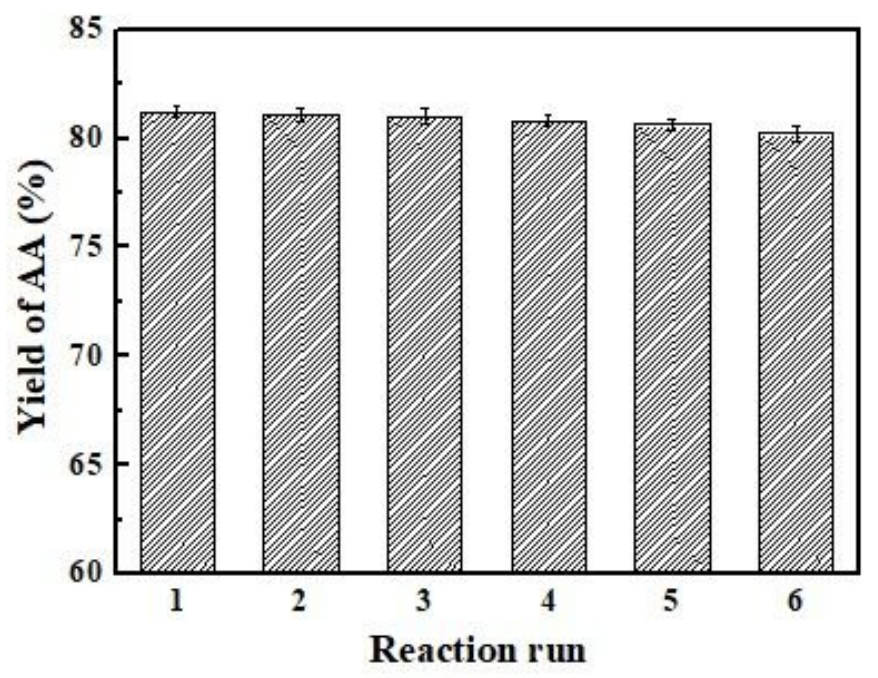

Figure 10

Recyclability of 31\%CSPW@MIL-101 catalysts

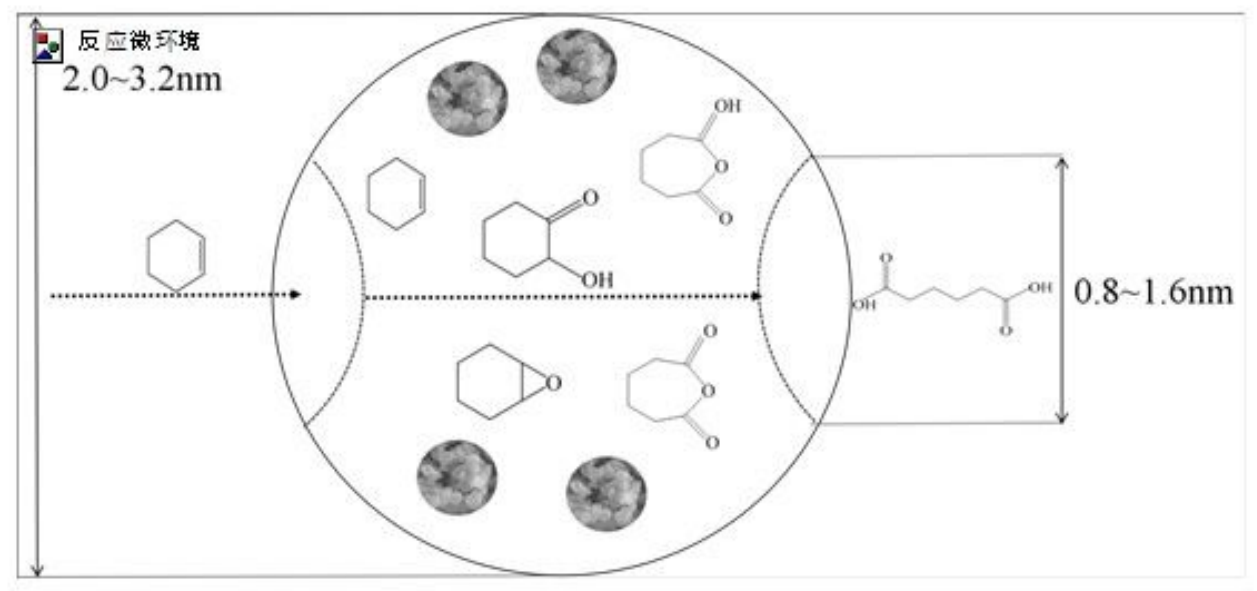

Figure 11

Schematic diagram of reaction mechanism in X\%CSPW@MIL-101 catalyst

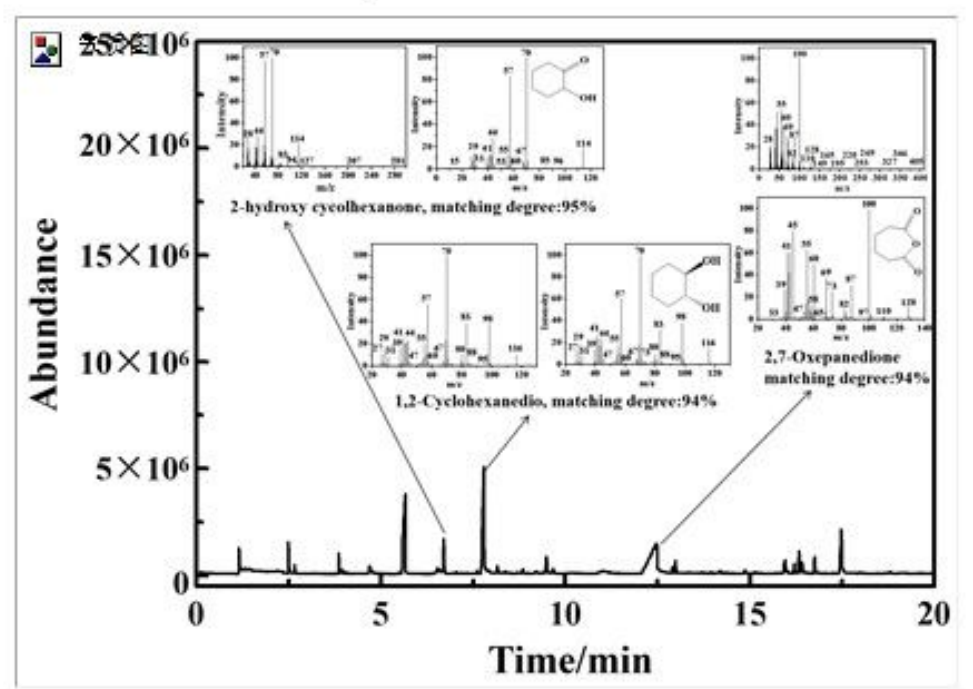


Figure 12

Gas chromatography mass spectrometry of reaction solution

\section{Supplementary Files}

This is a list of supplementary files associated with this preprint. Click to download.

- Highlights.doc

- GraphicalAbstract.doc

- Scheme1.jpg 